\title{
Automated Manufacturing Technology
}

\author{
Yingshu Sun, Songlin Duan
}

School of mechanical and electrical engineering, Shandong University of science and technology, Qingdao 266590, PR China

\begin{abstract}
Advanced manufacturing technology (advanced manufacturing technique, acronym AMT, specifically refers to the technology equipment and system which are produced by the integration of mechanical engineering technology electronic technology automation technology information technology and so on. Mainly include: computer-aided design, computer-aided manufacturing, integrated manufacturing systems. Advanced manufacturing technology is not a process method of a single processing process, but it spans many disciplines and encompasses all the relevant technologies throughout the entire product life cycle, from product design, processing and manufacturing to product sales and customer service. Involved in design, process, processing automation, management and special processing and other areas, and gradually integration and integration. And advanced manufacturing technology mainly includes the following three technology groups: (1) Main technology group: is the core of manufacturing technology, it includes two basic parts: related product design technology and process technology. (2) Support technology group: A. Information technology: interface and communication, database technology, integration framework, software engineering artificial intelligence, expert system and neural network, decision support system. Standards and frameworks: data standards, product definition standards, process standards, inspection standards, interface frameworks. C. Machine tools and tools technology. D. D. Sensor and control technology: single machining unit and process control, actuator, sensor and sensor combination, production scheduling. Others; (3) Manufacturing technology infrastructure. Elements include training and education of workshop workers, engineers and managers in various advanced production technologies and programs.
\end{abstract}

KEYWORDS: Manufacturing, Computer aided Design and Manufacturing, Computer Integrated Manufacturing, Flexible Manufacturing, numerical control technique

\section{Introduction}

Advanced manufacturing technology is based on the traditional manufacturing, continuously absorbs the achievements of machinery, electronics, information, materials, energy and modern management technology, and applies it to product 
design, manufacturing, testing, management, sales and use. The whole manufacturing process of service, in order to achieve high quality, high efficiency, low consumption, clean and flexible production, improve the adaptability and competitiveness of dynamic market, It is also the general name of the manufacturing technology that obtains the ideal technical economic benefit. Advanced manufacturing technology is not a general single-finger processing process, but across a number of disciplines, including from product design, processing and manufacturing To the whole life cycle of product sales, customer service and all related technologies, including design, process, processing automation, management and special processing and other areas, and gradually integration and integration.

Advanced manufacturing technology is the focus of international scientific and technological competition. With the development of society and the individuation and diversification of market demand, people's demand for products is becoming more and more diversified, and the market competition is becoming increasingly fierce. In order to survive and develop in the increasingly fierce market competition, enterprises must adopt advanced manufacturing technology.

\section{Computer aided Design and Manufacturing Technology}

\subsection{The concept of CAPP}

Computer aided process planning (CAPP) has completely changed the backwardness of traditional manual design, and it plays an extremely important role in modern automation integrated manufacturing system. The research of CAPP system mode and CAPP technology is an important task of manufacturing industry. This paper summarizes the concept and classification of CAPP system, summarizes the development trend and existing problems of CAPP, and summarizes the application of CAPP system in information construction of manufacturing industry in detail.

Computer aided process Planning (CAPP: Computer-aided process planning) refers to the conversion of the design information of parts into a series of processing operations required for manufacturing parts according to the constraints and resource conditions in the system composed of human and computer. The machine and machine interactively or automatically generate the machining process plan of the parts, that is, the process of matching and optimizing the product design information with all the possible machining capability information provided by the manufacturing environment [1]. Traditionally, CAPP is narrow sense, it is only for parts, mainly the design of machining technology, and It is often at the workshop level. Later gradually expanded to a variety of professional process design and even process information management.

Up to now, the research on CAPP has been carried out for more than 40 years, and many important research results have been obtained, and some economic benefits have been obtained. Especially, artificial intelligence technologies such as expert system, neural network and fuzzy logic have been applied to CAPP. The 
efficiency and flexibility of process knowledge acquisition, expression and application have been greatly improved, and the practical and engineering level of CAPP system has been greatly improved.

\subsection{Application of CAPP system in Manufacturing Industry}

CAPP system is the bridge of information design, manufacture and production. It links production management with product design. With the introduction of CAPP system, the manufacturing industry can effectively improve the working efficiency of production and manufacture, reduce the production cost, and push the enterprise to the road of information development. The application of CAPP system in manufacturing industry is as follows.

(1) CAPP system can realize the goal of exchanging information with CAM. This is mainly through the CAPP system to produce, machining parts necessary processing equipment, cutting parameters, clamping parameters, tooling equipment, plus parts in the cutting process of the tool path file transfer to the CAM system, And can accept the modification information from CAM system at any time.

(2) CAPP system can realize the goal of exchanging information with CAD. From the angle of dependency, CAD contains the CAPP system. According to the information of structure, material parameter, precision, precision and geometry in CAD, the CAPP system [8] is produced. CAD is the original input source of CAPP system. And it can transmit the structure process evaluation information to CAD.

(3) CAPP system can exchange information with MIS. CAPP system can transmit the information of equipment, tooling, man-hour and production route to MIS.. You can also receive information from MIS on equipment changes such as tool holder, raw material inventory and preparation plan.

(4) CAPP system can exchange information with quality assurance system. CAPP system can transmit process parameters such as production equipment, tooling and working procedure to CAQ. by using quality inspection rules and quality control plan. And receive the rework information of CAQ transmission.

(5) CAPP system can exchange information with manufacturing automation. CAPP system provides a series of information such as each process file, tool and fixture to MAS.. And real-time receive MAS transmission of technical modification and props use report.

(6) CAPP system can extract ERP directly from the process database of its own system, or transmit process parameters to these systems. 


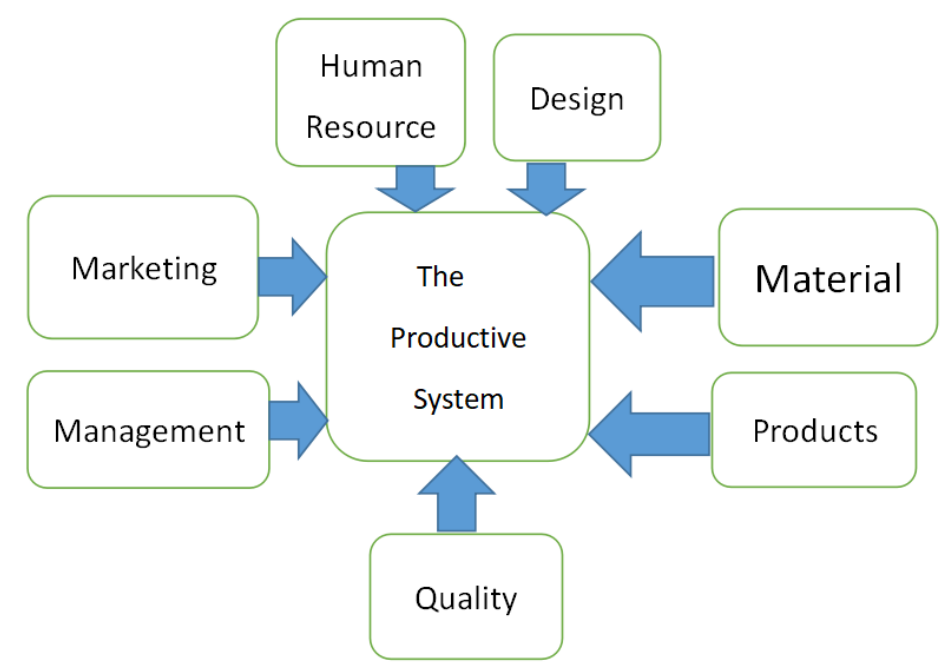

Figure. 1 A PACS Working in a Global Manufacturing System

\subsection{Typical CAPACS in manufacturing}

Fig.2 gives an overview of interrelated functions of CAPACS working from an integrated database system. Typical CAPACS in manufacturing are as follows: CAD, CAIN, CAM, CAPP, CAQC, CIPM, DCN, and GT.

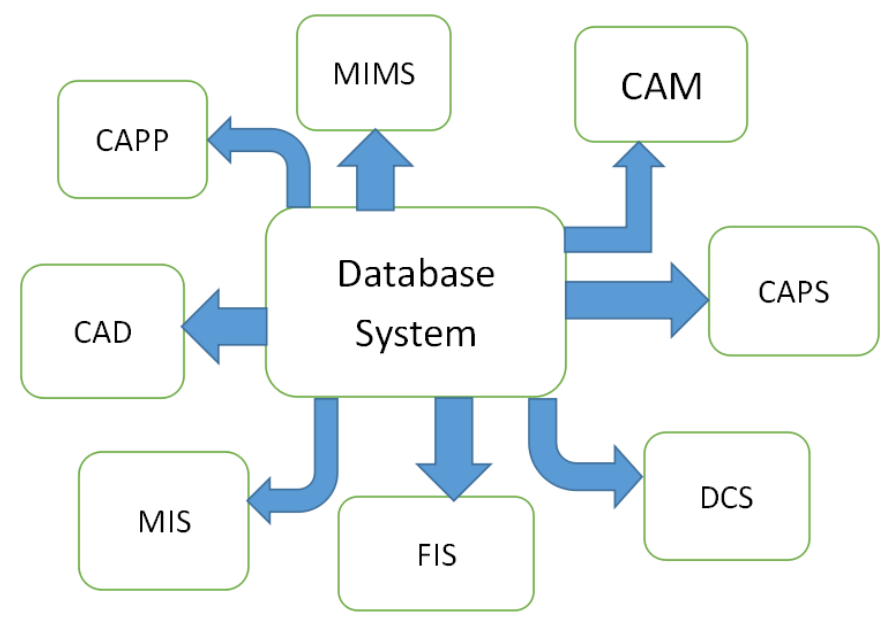

Figure. 2 Interaction of CAPACS in a Manufacturing System 


\section{Computer Integrated Manufacturing}

Computer integrated manufacturing is the term used to describe the modern approach to manufacturing. Modern manufacturing encompasses all of the activities and processes necessary to convert raw materials into finished products, deliver them to the market, and support them in the field.

CIM haves many benefits. (1) Product quality increases. (2) Lead times are reduced. (3) Direct labor costs are reduced. (4) Product development times are reduced. (5) Inventories are reduce. (6) Overall productivity increases. (7) Design quality increases.

\subsection{Composition of computer integrated manufacturing system}

The main technical foundation of computer integrated manufacturing system is flexible manufacturing system, which is integrated flexible manufacturing system. As a complex system integration, the computer integrated manufacturing system must be hierarchical. it is generally divided into five layers: (1) the first layer is the factory layer, which is the highest level of the overall resources, production activities and management of the decision-making plant. (2) the second layer is the workshop layer, which is also called the interval layer. The workshop here is not the "workshop" concept in the factory at present. The workshop layer only means that it should perform some functions in the whole activity of the factory, and carry out the resource allocation and task management. (III) Three layers are cell layers that govern the processing or assembly of a product. (4) the fourth layer is the workstation layer, which will coordinate a set of equipment within the station. (5) the fifth layer is the equipment layer, which is some specific equipment, such as machine tool, measuring machine, etc., which will perform specific processing, assembly or measuring tasks.

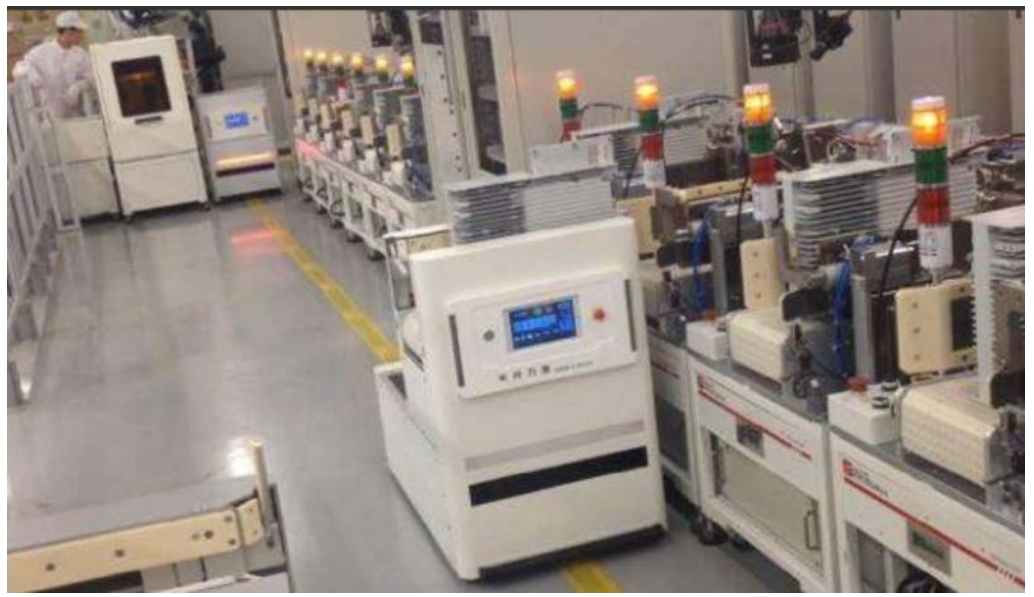

Figure. 3 Computer Integrated Manufacturing System 
A computer integrated manufacturing system based on the above hierarchy principle is generally considered to consist of a management information system, a computer aided engineering system, a production process control and management system, and material storage. A large system consisting of four subsystems and a database.

\subsection{Three main Features of computer Integrated Manufacturing system}

(1). Computer Integrated system with Information Integration as its main feature

From the 1970s to the 1980s, with sophisticated production as the organizational principle, a series of products were produced, automation equipment and systems were introduced, total quality management was realized, liquidity was reduced, and the turnover of funds was increased. It is also widely used in CAD, CAM robot.

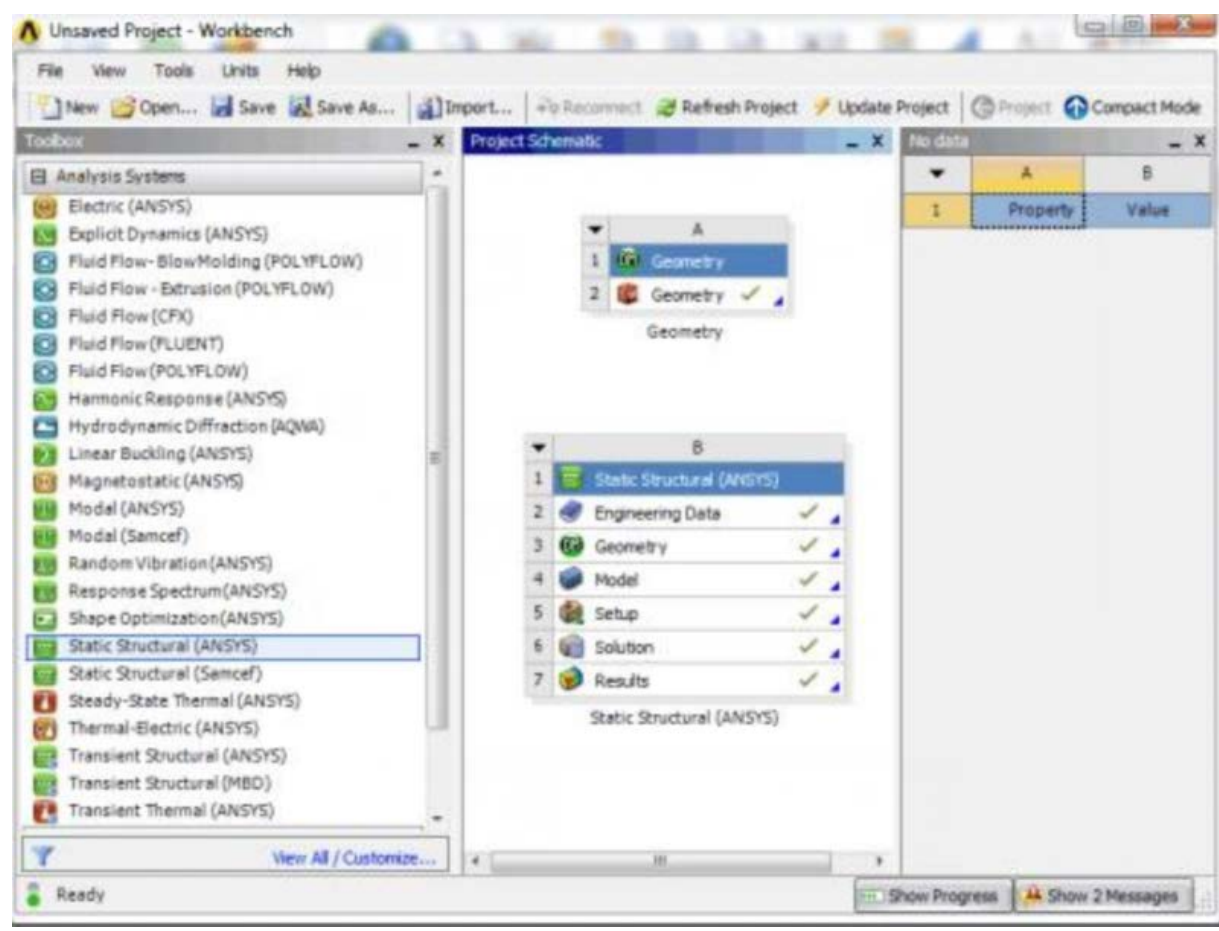

Figure. 4 Computer Integrated system

(2). Computer Integration system with process Integration as its main feature

It requires product developers to consider all factors throughout the product life cycle from concept formation to end-of-life handling including quality cost speed planning and user requirements at the beginning of the design. It emphasizes the 
team work spirit and working style, emphasizes the systematisms of the design process, emphasizes the parallelism of the design process, and emphasizes the quickness of the design process.

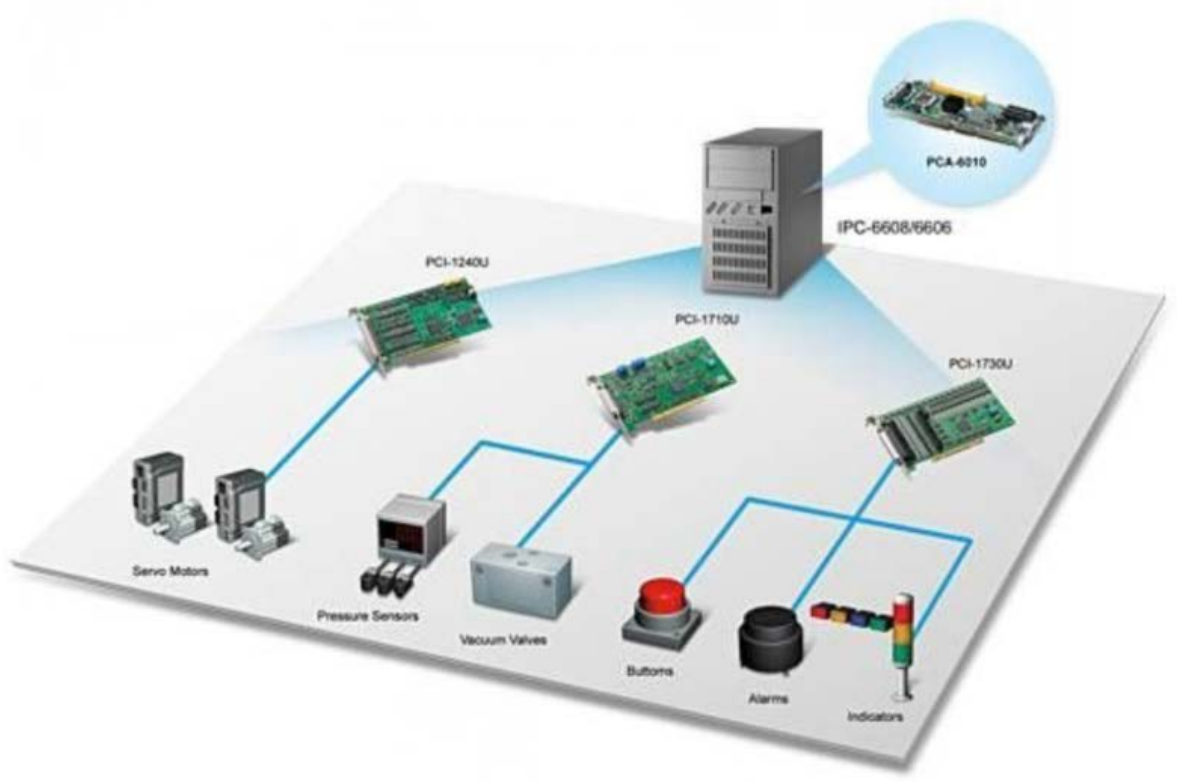

Figure. 5 Computer Integration system with process Integration as its main feature

(3) Computer integrated manufacturing system with enterprise integration as its main feature

Agile manufacturing emphasizes the use of flexible, advanced and practical manufacturing technology, proficiency in production skills, the realization of highquality workers, between enterprises and the internal flexible management of the organic combination. To achieve overall optimization and rapid response to the market. Agile manufacturing enterprises emphasize enterprise alliance, and compared with concurrent engineering stage, agile manufacturing enterprises have made further progress. 


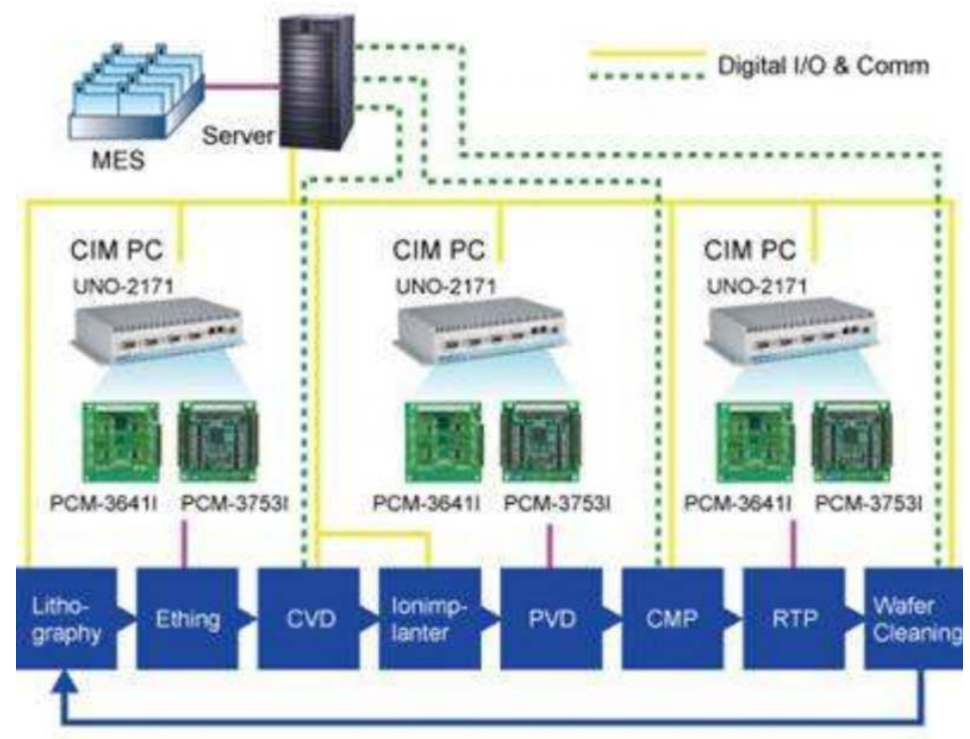

Figure. 6 Computer integrated manufacturing system with enterprise integration as its main feature

\subsection{Application of computer integrated manufacturing system}

This is the highest level of production system, is the soul of enterprises, it will make strategic decisions and macro management of production. According to the information of market demand and material supply, it determines the investment strategy and production plan through the decision model from the view of the overall situation and the long term. At the same time, the information and data of the decision result are connected and exchanged with each subsystem through database and communication network, and each subsystem is managed.

\section{Flexible Manufacturing}

With the development of economy and the improvement of consumption level, people pay more attention to the continuous renewal and diversification of products. Medium and small batch production and multi-variety production have become an important feature of the machinery manufacturing industry.

In the modern manufacturing setting, flexibility is an important characteristic.It means that a manufacturing system is versatile and adaptable, while also capable of handling relatively high production runs. 


\subsection{Flexible manufacturing can be divided into four categories according to its size}

(1). Flexible manufacturing unit

The flexible manufacturing unit is made up of 1 or 2 machining centers, industrial robots, NC machine tools and storage equipment for material transportation. It has the flexibility to process a variety of products.

\section{(2). Flexible manufacturing system}

It usually includes 4 or more automatic NC machine tools (machining center and turning center etc.), which is connected by centralized control system and material handling system, and can be processed and treated in many varieties, medium and small batches without downtime.

(3). Flexible manufacturing line

It is a production line between single or small batch non-flexible automatic line and medium-and small-batch multi-variety fms. The processing equipment can be a general machining center, cnc machine tool, or a special machine tool or machine tool. The requirement of material handling system flexibility is lower than that of fms, but the productivity is higher. It is represented by the flexible manufacturing system in discrete production and the decentralized control system (dcs) in the continuous production process. Its characteristic is to realize the flexibility and automation of production line. The technology has matured day by day and has entered the practical stage so far.

\section{(4). Flexible manufacturing plant (fmf)}

Fmf is a complete fms. That connects multiple fms, is matched by automatic three-dimensional warehouse, connects with computer system, and uses complete fms. From ordering, designing, processing, assembling, inspecting and transporting to delivery. It includes cad/cam, and makes the computer integrated manufacturing system (cims) put into practice, realizes the flexibility and automation of the production system, and then realizes the production management of the whole factory. Fmf is the highest level of automatic production, reflecting the most advanced automation application technology in the world. It is the automation of manufacturing, product development and management. The intelligent manufacturing system (ims), which controls the flow of information, is characterized by the flexibility and automation of the factory.

\subsection{Key Technologies of flexible Manufacturing system}

(1). Computer aided design

(2). Fuzzy control technology

(3). Artificial intelligence, expert system and intelligent sensor technology. 


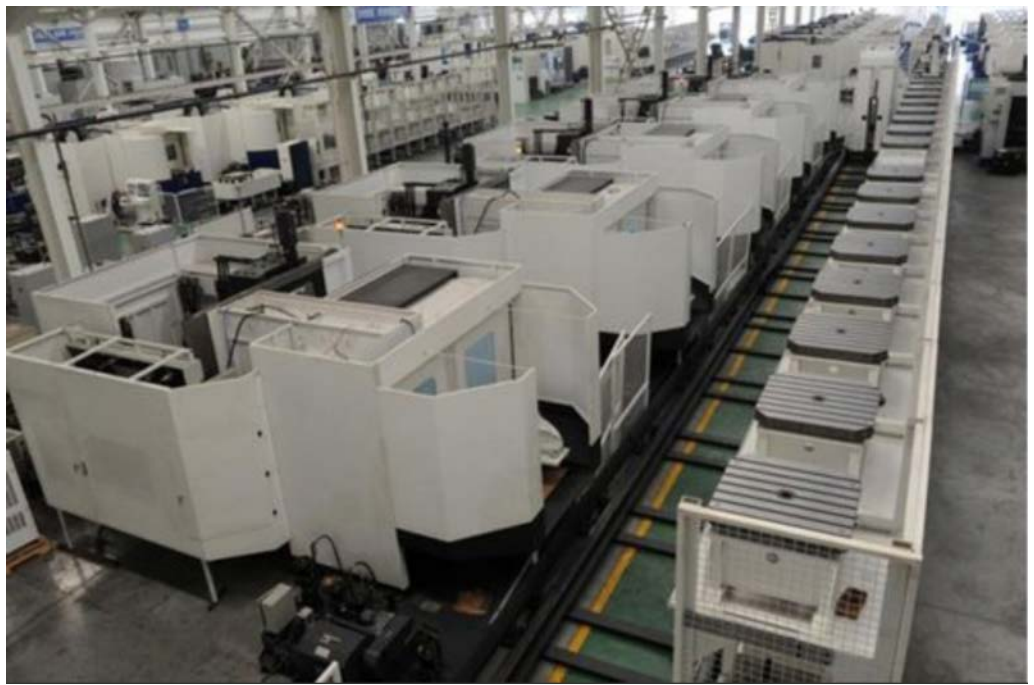

Figure. 7 Modern equipment for flexible manufacturing systems

\subsection{Development trend of flexible Manufacturing system}

(1) Fmc will become a popular Technology in Development and Application

This is because the investment of fmc is much smaller than that of fms, and the economic benefits are close, so it is more suitable for small and medium-sized enterprises with limited financial resources. At present, many foreign manufacturers will fmc as a heavy development.

(2) Developing more efficient fml

The demand for fml from factories such as automobiles and tractors has attracted much attention from fms manufacturers. It is the development trend of fml to replace the general machining center with low price special NC machine tool.

(3) Towards multifunction

It is a novel concept mode and new development trend to realize the new concept mode and new development trend of the future factory to further develop the multifunction fms.fms which is developed by the pure processing fms to weld, assemble, inspect, and the sheet metal processing and even casting, forging and other manufacturing processes. It is a strategic move that determines the future development of manufacturing enterprises. 


\section{Numerical control technique}

Numerical control technology plays an important role in the economic construction of human society and is applied in many aspects of social and economic construction. Numerical control technology has become the social and economic construction and people's life can not be lack of technical support part. In the future society, numerical control technology also plays a very important role in the development of society. The development of China's social construction and the development of numerical control technology have greatly promoted the development of mechanical processing industry and obtained good economic benefits. From the development situation of mass production, the development situation of high-precision technology has been gradually turned to the development situation of high-precision technology.

\subsection{The concept of numerical Control Technology}

Numerical control technology is a digital programming to control the automatic operation of machinery or other equipment from the early NC (hardware numerical control) to the use of CNC (hardware and software numerical control). With the increasingly fierce competition in the market, the upgrading of products is speeding up, the number of mass products is becoming smaller and fewer, and the proportion of small batch products is becoming larger and larger. Therefore, a kind of high precision, flexible and good processing equipment is urgently needed to meet these needs. The rapid development of electronic technology and computer technology provides a solid technical foundation for the progress of NC machine tools. Numerical control technology was born and developed under this background. It came into being from Dynamic technology brings new concepts and promotes the development of processing automation technology.

\subsection{The Development status of numerical Control Technology}

Since the 1990s, with the rapid development of computer technology in the world, the latest technological achievements in the fields of computer and control theory have been continuously adopted in numerical control technology. In the last 30 years, the performance and function of CNC have been developing continuously, and the CNC machine tools have developed to the direction of comprehensive automation. Therefore, CNC machine tool technology, is considered to be the basic technology of modern mechanical automation.

In some highly sophisticated industries, numerical control technology is more widely used. For example, in the field of aviation and aerospace, data analysis is carried out almost entirely by computers. The superior performance of numerical control machine tools has the most obvious manifestation in this respect. The high precision of weight and size need computer to check repeatedly before production, otherwise it will cause huge casualties and economic losses. 
In the 21st century, with the continuous expansion of the industry, the domestic high-grade CNC machine tools have made remarkable progress, and the domestic high-grade numerical control system has made a major breakthrough, which fully shows that the overall level of China's CNC machine tools has been comprehensively improved. At the same time, the quality of CNC machine tools is developing rapidly in the process of catching up with the world. At the same time, as the core technology of CNC machine tool, domestic CNC system has made great breakthrough and progress in this change.

\subsection{Problems existing in the Development of numerical Control Technology in China}

There are still some problems in the modern production line of numerical control production in our country, which makes the CNC machine tools produced in our country unable to compare with those produced in Germany, Korea and other countries. To put it simply, large-scale machine tools in China have occupied a certain position in the world, but from the service life of the machine tools, we can see that there are still problems in the CNC machine tools produced in our country. And in some specialized instruments that require very high precision, machine tools cannot be manufactured in batches in our country at present. Moreover, the production of machine tools in our country occupies a relatively large area and the appearance is not very satisfactory. Therefore, the state has allocated funds for continuous improvement in these areas. It is expected to catch up with the developed western countries in the last ten years Home CNC machine tool production technology. We also need to do a lot of work in software developers, to design numerical control software that conforms to the characteristics of different industries, and to create a CNC airport brand belonging to China according to the characteristics of different industries. In the future development, gradually catch up with the western developed countries in numerical control technology. As far as the analysis of China's current numerical control technology is concerned, it is still far from other countries' numerical control technology. Only by clearly understanding the development process of numerical control technology and the present situation of numerical control technology in our country, can the further development of numerical control technology be realized. So as to promote the rapid development of social and economic construction.

\section{Conclusion}

Advanced manufacturing technology is the latest development stage of manufacturing technology, and the technological manufacturing industry facing the 21st century is the guarantee of social material civilization and dynamic development with human society. Manufacturing technology will inevitably be updated with the progress of science and technology. Advanced manufacturing technology is the latest stage of development of manufacturing technology. It is derived from traditional manufacturing technology and maintains the effective 
elements of manufacturing technology in the past. However, with the infiltration of high and new technology and the change of manufacturing environment, there has been a change in quality. Advanced manufacturing technology is a complete group of technologies that combine manufacturing technology with modern high and new technology. Category of the new technical field, is oriented to the 21 st century of technology.

Advanced manufacturing technology is not inflexible, but is a dynamic process. We must constantly absorb all kinds of high-tech achievements and infiltrate them into all areas and processes of product design, manufacturing, production management and marketing. And achieve high quality, high efficiency, low consumption, clean production.

China's manufacturing industry is facing more and fiercer competition, innovation has become the focus of enterprise competition strategy, the new production model attaches more and more importance to knowledge, and we must adopt advanced manufacturing technology to transform the traditional industry. However, in our country, the application of advanced manufacturing technology and the development of advanced production models in the manufacturing industry must be based on the national conditions of our country, on the basis of tracking and digesting foreign advanced production models, and on the basis of the national conditions. To develop our advanced manufacturing production model and promote modern advanced manufacturing technology.

\section{References}

[1] Bray O H. 1992. Computer Integrated Manufacturing: the Data Management Strategy. New York: Digital Press.

[2] Dickens P M. 1995. Research developments in rapid prototyping. Pro Inst Eng, (209): 267-270.

[3] Joshi S B, Smith J S, 1994. Computer Control of Flexible Manufacturing System: Research and Development.London Chapman \& Hall.

[4] Kalpakjian S. 2001. Manufacturing Engineering and Technology. London: Prentice Hall.

[5] Koening D T.1987.Manufacturing Engineering Principles for Optimization. Berlin.

[6] Niebel B W. 1989. Modern Manufacturing Process Engineering. New York: McGraw-Hill.

[7] Nolen J.1989. Computer Automated Process Planning for World Class Manufacturing. London: M.Dekker.

[8] Steven L, Goldman R N, 1997. Agile Competitors and Virtual Organizations. New York: Van Nostrand Reinhold Publishing Inc.

[9] Zhang H C, Kuo T C, Lu H T.1997. Environmentally conscious design and manufacturing. Journey of Manufacturing System, 16 (5): 123-126. 